\title{
Conics and Quadrics in Lorentzian Space
}

\author{
Ayşe Çiçek Gözütok, Sıddıka Özkaldı Karakuş* and Halit Gündoğan
}

(Communicated by Bülent ALTUNKAYA)

\section{Abstract}

In this paper we give geometric definitions of the Lorentzian ellipse, Lorentzian hyperbola, and Lorentzian parabola as plane curves in Lorentzian plane. Moreover we obtain the equations of Lorentzian revolution ellipsoid, Lorentzian revolution hyperboloid and Lorentzian revolution paraboloid respectively.

Keywords: Conics; quadrics; Lorentzian space.

AMS Subject Classification (2010): Primary: 53B30 ; Secondary: 51N10; 53A04

${ }^{*}$ Corresponding author
\end{abstract}

\section{Introduction}

In Euclidean 2-space $\mathbb{R}^{2}$, the graph of the equation $y=a x^{2}+b x+c$ is a curve called a parabola. It is given general definitions for this and two closely related curves called, respectively, the ellipse and hyperbola. These curves, the ellipse, hyperbola, and parabola, have been known and studied by mathematicians since ancient times long before they were identified as graphs of second degree equations. Collectively, along with circles and certain combinations of two straight lines, they are called the conics or conic sections, because they were first studied by the ancient Greeks as the curves of intersection of a plane with a cone. These standart equations are special cases of the general equation of second degree in $x$ and $y$; that is, they are special cases of the equation

$$
A x^{2}+B x y+C y^{2}+D x+E y+F=0,
$$

in which some of the coefficients will be zero. The cone can be tought of as the surface generated by rotating a line $l$ about a fixed line $l^{\prime}$ called the axis of the cone, in such a way that the angle between $l$ and $l^{\prime}$ remains constant and is not zero. The point $V$ in which the lines intersect is called the vertex of the cone. Any line through $V$ lying in the surface is called a generating line or a generator of the cone. The cone consists of two parts or nappes having only the vertex in common. If a plane, not containing the vertex and not parallel to a generator, intersects both nappes of the cone, the curve of intersection is a hyperbola, a curve consisting of two disconnected branches. If such a plane intersects only one nappe, the curve of intersection is an ellipse. If the plane is perpendicular to the axis of cone, the ellipse becomes a circle. If the plane is parallel to a generator, the curve of intersection is a parabola. It is clear that a plane through the vertex can intersect the cone either in the single point $V$, in two intersecting straight lines through $V$, or in a single generating line if the plane is tangent to the cone.

But, to our knowledge, there has been no study on the conics and quadrics in Lorentzian space. In this paper,we shall not adopt this historic point of view in discussing the conics. Instead we give geometric definitions of the Lorentzian ellipse, Lorentzian hyperbola, and Lorentzian parabola as plane curves in Lorentzian plane. Moreover we obtain the equations of Lorentzian revolution ellipsoid, Lorentzian revolution hyperboloid and Lorentzian revolution paraboloid respectively.

But before this, we will survey briefly the fundamental concepts and properties in the Lorentzian space. We refer mainly to O'Neill and Ratcliffe [1,3].

Received : 25-10-2017, Accepted : 03-04-2018 
Let $\mathbb{R}_{1}^{2}$ be the 2-dimensional the Lorentzian space with Lorentzian metric $<_{,}>_{L}: d x_{1}^{2}-d x_{2}^{2}$ and let $\mathbb{R}_{1}^{3}$ be the 3-dimensional the Lorentzian space with Lorentzian metric $<,>_{L}: d x_{1}^{2}+d x_{2}^{2}-d x_{3}^{2}$. It has been known that in $\mathbb{R}_{1}^{3}$ there are three categories of curves and vectors, namely, spacelike, timelike and null, depending on their causal character. Let $\vec{x}$ be a tangent vector of Lorentzian space. Then $\vec{x}$ is said to be spacelike if $\langle\vec{x}, \vec{x}\rangle>0$ or $\vec{x}=\overrightarrow{0}$, timelike if $\langle\vec{x}, \vec{x}\rangle\left\langle 0\right.$, null (lightlike) if $\langle\vec{x}, \vec{x}\rangle=0$ and $\vec{x} \neq \overrightarrow{0}$. Let $\alpha: I \subset \mathbb{R} \longrightarrow \mathbb{R}_{1}^{3}$ be a regular curve in $\mathbb{R}_{1}^{3}$. Then, the curve $\alpha$ is spacelike if all its velocity vectors are spacelike. Similarly, it is called timelike and null curve if all its velocity vectors are timelike and null vectors, respectively.

\section{The Conic In Lorentzian Plane $\mathbb{R}_{1}^{2}$}

In this section we shall study three curves that belong together from several points view. These are the Lorentzian ellipse, Lorentzian hyperbola, and Lorentzian parabola; they are called the Lorentzian conic section curves, or Lorentzian conic sections. Our approach will be to define each curve by a geometric property, obtain an analytic representation for the curve.

\subsection{The Lorentzian Ellipse}

We assume that all points considered lie in the Lorentzian plane $\varepsilon_{2}$. Let $F_{1}$ and $F_{2}$ be points and the length of the segment $F_{1} F_{2}$ be denoted by $2 c$. If $a$ is any number greater than $c$, the set points $P$ such that $d\left(F_{1}, P\right)+d\left(F_{2}, P\right)=2 a$ is called Lorentzian ellipse.

Definition 2.1. A Lorentzian ellipse is a set of points $P$ in the Lorentzian plane such that the sum of the distances of $P$ from two fixed points $F_{1}$ and $F_{2}$ is constant. Each of the points $F_{1}$ and $F_{1}$ is called a focus of the Lorentzian ellipse. The plural form of focus is foci.

Choose a coordinate system with the line through $F_{1}$ and $F_{2}$ as $x$-axis and perpendicular bisector of the segment $F_{1} F_{2}$ as $y$-axis. If $d\left(F_{1}, F_{2}\right)=2 c$, the coordinates of the foci are $F_{1}(c, 0)$ and $F_{2}(-c, 0)$. In this case the foci are on the spacelike axis. If $P(x, y)$ is any point on the ellipse, the defining property

$$
d\left(F_{1}, P\right)+d\left(F_{2}, P\right)=2 a,
$$

can be written in the form

$$
\sqrt{(x-c)^{2}-y^{2}}+\sqrt{(x+c)^{2}-y^{2}}=2 a .
$$

It is clear that $d\left(F_{1}, P\right)+d\left(F_{2}, P\right) \geq d\left(F_{1}, F_{2}\right)$ and hence we must have $2 a \geq 2 c$. If $a=c, P$ could only lie on the line segment $F_{1} F_{2}$. This case is not interesting and will be excluded. We assume therefore that $a>c$. Equation (2.1) may be written

$$
\sqrt{(x-c)^{2}-y^{2}}=2 a-\sqrt{(x+c)^{2}-y^{2}} .
$$

Taking the square of each side and simplifying yields

$$
a \sqrt{(x+c)^{2}-y^{2}}=a^{2}+c x .
$$

Taking the square of each side again and simplifying we get

$$
\left(c^{2}-a^{2}\right) x^{2}+a^{2} y^{2}=-a^{2}\left(a^{2}-c^{2}\right) .
$$

Since $a>c, a^{2}-c^{2}$ is positive and there is a positive real number $b$ such that $b^{2}=a^{2}-c^{2}$. Equation (2.3) therefore takes the form

or, upon dividing by $a^{2} b^{2}$,

$$
b^{2} x^{2}-a^{2} y^{2}=a^{2} b^{2},
$$

$$
\frac{x^{2}}{a^{2}}-\frac{y^{2}}{b^{2}}=1
$$

We called equation (2.4) as the standart equation of the Lorentzian ellipse with center at the origin and foci on the spacelike $x$-axis.

If the foci are $F_{1}(0, c)$ and $F_{2}(0,-c)$, which are on the timelike axis, then the standart equation of the Lorentzian ellipse with center at the origin and foci on the timelike $y$-axis, can be easily found

$$
-\frac{x^{2}}{b^{2}}+\frac{y^{2}}{a^{2}}=1 \text {. }
$$

Thus we give the following theorem: 
Theorem 2.1. A point $P(x, y)$ is on the Lorentzian ellipse determined by the number $a>0$ and the foci $F_{1}(c, 0)$ and $F_{2}(-c, 0)$, which are on the spacelike axis (or respectively $F_{1}(0, c)$ and $F_{2}(0,-c)$, which are on the timelike axis) if and only if $\frac{x^{2}}{a^{2}}-\frac{y^{2}}{b^{2}}=1$ (or respectively $-\frac{x^{2}}{b^{2}}+\frac{y^{2}}{a^{2}}=1$ ), where $a, b$, and c satisfy $a^{2}=b^{2}+c^{2}$.

\subsection{The Lorentzian Hyperbola}

The definition and many of the properties of the hyperbola are so closely analogous to the ellipse. Let two points $F_{1}$ and $F_{2}$ be given and their distance apart be denoted by $2 c$. If $0<a<c$, the set of points $P$ for which $\left|d\left(F_{1}, P\right)+d\left(F_{2}, P\right)\right|=2 a$ is called a Lorentzian hyperbola.

Definition 2.2. A Lorentzian hyperbola is a set of points $P$ in the Lorentzian plane such that the difference of the distances of $P$ from two fixed points $F_{1}$ and $F_{2}$ is constant. Each of the points $F_{1}$ and $F_{1}$ is called a focus of the Lorentzian hyperbola.

Choose coordinate axes as for the hyperbola with the line through $F_{1} F_{2}$ for $x$-axis and perpendicular bisector of $F_{1} F_{2}$ for $y$-axis. If $d\left(F_{1}, F_{2}\right)=2 c$, the coordinates of the foci are $F_{1}(c, 0)$ and $F_{2}(-c, 0)$. In this case the foci are on the spacelike axis. Then if $P(x, y)$ is any point on the hyperbola, the defining property

$$
\left|d\left(F_{1}, P\right)-d\left(F_{2}, P\right)\right|=2 a,
$$

can be written in the form

$$
\sqrt{(x-c)^{2}-y^{2}}-\sqrt{(x+c)^{2}-y^{2}}= \pm 2 a .
$$

By transposing one radical, taking the square, simplifying, and taking the square of each side again we get the equation

$$
\left(c^{2}-a^{2}\right) x^{2}+a^{2} y^{2}=-a^{2}\left(a^{2}-c^{2}\right)
$$

Since $a<c$ we can replace $c^{2}-a^{2}$ by $b^{2}$ and this equation becomes

$$
b^{2} x^{2}+a^{2} y^{2}=a^{2} b^{2},
$$

or, upon dividing by $a^{2} b^{2}$,

$$
\frac{x^{2}}{a^{2}}+\frac{y^{2}}{b^{2}}=1
$$

We called equation (2.6) as the standart equation of the Lorentzian hyperbola with center at the origin and foci on the spacelike $x$-axis.

If the foci are $F_{1}(0, c)$ and $F_{2}(0,-c)$, which are on the timelike axis, then the standart equation of the Lorentzian hyperbola with center at the origin and foci on the timelike $y$-axis, can be easily found

$$
\frac{x^{2}}{b^{2}}+\frac{y^{2}}{a^{2}}=1
$$

Thus we give the following theorem:

Theorem 2.2. A point $P(x, y)$ is on the Lorentzian hyperbola determined by the number $a>0$ and the foci $F_{1}(c, 0)$ and $F_{2}(-c, 0)$, which are on the spacelike axis (or respectively $F_{1}(0, c)$ and $F_{2}(0,-c)$, which are on the timelike axis) if and only if $\frac{x^{2}}{a^{2}}+\frac{y^{2}}{b^{2}}=1$ (or respectively $\frac{x^{2}}{b^{2}}+\frac{y^{2}}{a^{2}}=1$ ), where $a, b$, and c satisfy $a^{2}+b^{2}=c^{2}$.

\subsection{The Lorentzian Parabola}

The definition of a parabola involves distances from a point and a line.

Definition 2.3. Given a fixed point $F$, the focus, and a fixed line $l$, the directrix, a Lorentzian parabola is the set of points $P$ that are equidistant from $F$ and $l$.

Given a directrix $l$ and focus $F$, we choose for $x$-axis the line through $F$ perpendicular to $l$. If this line cuts $l$ in the point $D$, we find the simplest equation for the Lorentzian parabola results from choosing for origin the midpoint of the segment $D F$.

If we assume that the coordinates of $F$ are $(p, 0)$, which is on the spacelike axis, the equation of the directrix $l$ is then $x=-p$. The point $P(x, y)$ is then on the Lorentzian parabola if and only if $d(F, P)=d(M, P)$ where $M$ is the projection $P$ on $l$. This yields

$$
\sqrt{(x-p)^{2}-y^{2}}=|x+p|
$$


or taking the square of each side and simplifying,

$$
y^{2}=-4 p x,
$$

for an equation of the Lorentzian parabola relative to the chosen coordinate system.

In a similar vein, we note that we would have taken the focus $F$ on the $y$-axis at $F(0, p)$, which is on the timelike axis, and the directrix perpendicular to the $y$-axis, i.e., $y=-a$. This interchange of the roles of $x$ and $y$-axes would obviously produce the equation

$$
x^{2}=-4 p y .
$$

Thus we give the following theorem:

Theorem 2.3. A point $P(x, y)$ is on the Lorentzian parabola determined by the focus $F(0, p)$ (or respectively $F(p, 0)$ ) and the directrix $l: x=-p$ (or respectvely $y=-p$ ) if and only if $y^{2}=-4 p x$ (or respectively $x^{2}=-4 p y$ ).

\section{Revolution Quadric Surfaces in Lorentzian Space $\mathbb{R}_{1}^{3}$}

We conclude this study of surfaces with a description of some revolution quadric surfaces the three-dimensional analog of the conic section curves Lorentzian space.

From an analytic point view the relation between the conic section curves and the quadric surfaces is very strong: the former are the curves obtained from the general equation of second degree in $x$ and $y$; the latter are the surfaces represented by the general equation of second degree $x, y$, and $z$. As we shall see, there are close geometric ties between the two families. In particular the traces of the quadric surfaces in the coordinate planes are conic section curves.

As we know, the Lorentzian sphere which is the locus of a moving point remains at a constant distance from a fix point. The constant distance is the radius and the fixed point the center. The equation of a Lorentzian sphere and hyperbolic sphere of radius 1 in $\mathbb{R}_{1}^{3}$ are given by

$$
\mathbb{S}_{1}^{2}=\left\{X=\left(x_{1}, x_{2}, x_{3}\right):<X, X>_{L}=1\right\},
$$

and

respectively, [1].

Now we want to obtain the equations of Lorentzian revolution ellipsoid, Lorentzian revolution hyperboloid and Lorentzian revolution paraboloid respectively.

\subsection{The Lorentzian Revolution Ellipsoid}

We assume that all points considered lie in the Lorentzian space. Let $F_{1}$ and $F_{2}$ be points and the length of the segment $F_{1} F_{2}$ be denoted by $2 c$. If $a$ is any number greater than $c$, the set points $P$ such that $d\left(F_{1}, P\right)+d\left(F_{2}, P\right)=2 a$ is called Lorentzian revolation ellipsoid.

Definition 3.1. A Lorentzian revolution ellipsoid is a set of points $P$ in the Lorentzian space such that the sum of the distances of $P$ from two fixed points $F_{1}$ and $F_{2}$ is constant. Each of the points $F_{1}$ and $F_{1}$ is called a focus of the Lorentzian revolation ellipsoid.

If $d\left(F_{1}, F_{2}\right)=2 c$, the coordinates of the foci are $F_{1}(0, c, 0)$ and $F_{2}(0,-c, 0)$. In this case the foci are on the spacelike axis. If $P(x, y, z)$ is any point on the revolution ellipsoid, the defining property

$$
d\left(F_{1}, P\right)+d\left(F_{2}, P\right)=2 a,
$$

can be written in the form

$$
\sqrt{x^{2}+(y-c)^{2}-z^{2}}+\sqrt{x^{2}+(y+c)^{2}-z^{2}}=2 a .
$$

It is clear that $d\left(F_{1}, P\right)+d\left(F_{2}, P\right)>d\left(F_{1}, F_{2}\right)$ and hence we must have $a>c$. Equation (3.1) may be written

$$
\sqrt{x^{2}+(y-c)^{2}-z^{2}}=2 a-\sqrt{x^{2}+(y+c)^{2}-z^{2}} .
$$

Taking the square of each side and simplifying yields

$$
a \sqrt{x^{2}+(y+c)^{2}-z^{2}}=a^{2}+c y .
$$


Taking the square of each side again and simplifying we get

$$
a^{2} x^{2}+\left(a^{2}-c^{2}\right) y^{2}-a^{2} z^{2}=a^{2}\left(a^{2}-c^{2}\right) .
$$

Since $a>c, a^{2}-c^{2}$ is positive and there is a positive real number $b$ such that $b^{2}=a^{2}-c^{2}$. Equation (3.2) therefore takes the form

or, upon dividing by $a^{2} b^{2}$,

$$
a^{2} x^{2}+b^{2} y^{2}-a^{2} z^{2}=a^{2} b^{2}
$$

$$
\frac{x^{2}}{b^{2}}+\frac{y^{2}}{a^{2}}-\frac{z^{2}}{b^{2}}=1
$$

We called equation (3.4) as the standart equation of the Lorentzian revolution ellipsoid with center at the origin and foci on the spacelike axis.

If the foci are $F_{1}(0,0, c)$ and $F_{2}(0,0,-c)$, which are on the timelike axis, then the standart equation of the Lorentzian revolution ellipsoid with center at the origin and foci on the timelike axis, can be easily found

$$
-\frac{x^{2}}{b^{2}}+\frac{y^{2}}{a^{2}}+\frac{z^{2}}{b^{2}}=1
$$

Thus we give the following theorem:

Theorem 3.1. A point $P(x, y, z)$ is on the Lorentzian revolution ellipsoid determined by the number $a>0$ and the foci $F_{1}(0, c, 0)$ and $F_{2}(0,-c, 0)$, which are on the spacelike axis (or respectively $F_{1}(0,0, c)$ and $F_{2}(0,0,-c)$, which are on the timelike axis) if and only if $\frac{x^{2}}{b^{2}}+\frac{y^{2}}{a^{2}}-\frac{z^{2}}{b^{2}}=1$ (or respectively $-\frac{x^{2}}{b^{2}}+\frac{y^{2}}{a^{2}}+\frac{z^{2}}{b^{2}}=1$ ), where $a, b$, and c satisfy $a^{2}=b^{2}+c^{2}$.

\subsection{The Lorentzian Revolution Hyperboloid}

Let two points $F_{1}$ and $F_{2}$ be given and their distance apart be denoted by $2 c$. If $0<a<c$, the set of points $P$ for which $\left|d\left(F_{1}, P\right)+d\left(F_{2}, P\right)\right|=2 a$ is called a Lorentzian revolution hyperboloid.

Definition 3.2. A Lorentzian revolution hyperboloid is a set of points $P$ in the Lorentzian space such that the difference of the distances of $P$ from two fixed points $F_{1}$ and $F_{2}$ is constant. Each of the points $F_{1}$ and $F_{1}$ is called a focus of the Lorentzian revolution hyperboloid.

If $d\left(F_{1}, F_{2}\right)=2 c$, the coordinates of the foci are $F_{1}(0, c, 0)$ and $F_{2}(0,-c, 0)$. In this case the foci are on the spacelike axis. Then if $P(x, y, z)$ is any point on the revolution hyperboloid, the defining property

$$
\left|d\left(F_{1}, P\right)-d\left(F_{2}, P\right)\right|=2 a,
$$

can be written in the form

$$
\sqrt{x^{2}+(y-c)^{2}-z^{2}}-\sqrt{x^{2}+(y+c)^{2}-z^{2}}= \pm 2 a .
$$

By transposing one radical, taking the square, simplifying, and taking the square of each side again we get the equation

$$
a^{2} x^{2}-\left(c^{2}-a^{2}\right) y^{2}-a^{2} z^{2}=-a^{2}\left(c^{2}-a^{2}\right) .
$$

Since $a<c$ we can replace $c^{2}-a^{2}$ by $b^{2}$ and this equation becomes

$$
a^{2} x^{2}-b^{2} y^{2}-a^{2} z^{2}=-a^{2} b^{2},
$$

or, upon dividing by $-a^{2} b^{2}$,

$$
-\frac{x^{2}}{b^{2}}+\frac{y^{2}}{a^{2}}+\frac{z^{2}}{a^{2}}=1
$$

We called equation (3.6) as the standart equation of the Lorentzian revolution hyperboloid with center at the origin and foci on the spacelike axis.

If the foci are $F_{1}(0,0, c)$ and $F_{2}(0,0,-c)$, which are on the timelike axis, then the standart equation of the Lorentzian revolution hyperboloid with center at the origin and foci on the timelike axis, can be easily found

$$
\frac{x^{2}}{b^{2}}+\frac{y^{2}}{b^{2}}+\frac{z^{2}}{a^{2}}=1
$$

Thus we give the following theorem:

Theorem 3.2. A point $P(x, y, z)$ is on the Lorentzian revolution hyperboloid determined by the number $a>0$ and the foci $F_{1}(0, c, 0)$ and $F_{2}(0,-c, 0)$, which are on the spacelike axis (or respectively $F_{1}(0,0, c)$ and $F_{2}(0,0,-c)$, which are on the timelike axis) if and only if $-\frac{x^{2}}{b^{2}}+\frac{y^{2}}{a^{2}}+\frac{z^{2}}{a^{2}}=1$ (or respectively $\frac{x^{2}}{b^{2}}+\frac{y^{2}}{b^{2}}+\frac{z^{2}}{a^{2}}=1$ ), where $a, b$, and c satisfy $a^{2}+b^{2}=c^{2}$. 


\subsection{The Lorentzian Revolution Paraboloid}

The definition of a revolution paraboloid involves distances from a point and a line in the Lorentzian space.

Definition 3.3. Given a fixed point $F$, the focus, and a fixed line $l$, the directrix, a Lorentzian revolution paraboloid is the set of points $P$ that are equidistant from $F$ and $l$.

If we assume that the coordinates of $F$ are $(0, p, 0)$, which is on the spacelike axis, the equation of the directrix $l$ is then $y=-p$. The point $P(x, y, z)$ is then on the Lorentzian revolution paraboloid if and only if $d(F, P)=d(M, P)$ where $M$ is the projection $P$ on $l$. This yields

$$
\sqrt{x^{2}+(y-p)^{2}-z^{2}}=|y+p|
$$

or taking the square of each side and simplifying,

$$
x^{2}-z^{2}=4 p y
$$

for an equation of the Lorentzian revolution paraboloid relative to the choosen coordinate system.

In a similar vein, we note that we would have taken the focus $F(0,0, p)$, which is on the timelike axis, and the directrix perpendicular to the $z$-axis, i.e., $z=-p$. Thus we can get easily,

$$
x^{2}+y^{2}=-4 p y .
$$

Thus we give the following theorem:

Theorem 3.3. A point $P(x, y, z)$ is on the Lorentzian revolution paraboloid determined by the focus $F(0, p, 0)$ (or respectively $F(0,0, p)$ ) and the directrix $l: y=-p$ (or respectvely $z=-p$ ) if and only if $x^{2}-z^{2}=4 p y$ (or respectively $x^{2}+y^{2}=-4 p y$ ).

\section{Acknowledgement}

We wish to thank the referee for the careful reading of the manuscript and constructive comments that have substantially improved the presentation of the paper.

\section{References}

[1] O'Neill, B., Semi-Riemannian Geometry With Applications to Relativity, Academic Press, Inc, ork, 1983

[2] Protter M.H., Analytic Geometry, Addison-Wesley Publishing Company, 1975

[3] Ratcliffe, J.G.Foundatitons of Hyperbolic Manifolds, Springer-Verlag, New York, 1994

[4] Smith W.K, Analytic Geometry, The Macmillan Company, New York, 1972.

\section{Affiliations}

AYŞE Ç. GÖZÜTOK

AdDRESS: Abant Izzet Baysal University, Electronics and Automation, Bolu Vocational School, Bolu, Turkey E-MAIL: aysegozutok@ibu.edu.tr

ORCID ID: $0000-0002-9470-5196$

SIDDIKA ÖZKALDI KARAKUŞ

ADDRESS: Bilecik Şeyh Edebali University,Department of Mathematics, Faculty of Science and Arts, Bilecik, Turkey. E-MAIL: siddika.karakus@bilecik.edu.tr

ORCID ID: $0000-0002-2699-4109$

HALIT GÜNDOĞAN

AdDress: University of Kırıkkale, Department of Mathematics, Faculty of Science and Arts, 71450-Yahşihan, Kırıkkale, Turkey

E-MAIL: hagundogan@hotmail.com

ORCID ID: $0000-0002-5806-2090$ 